\title{
Behavioral Health Screening and Care Coordination for Rural Veterans in a Federally Qualified Health Center
}

\author{
M. Bryant Howren, PhD, MPH \\ Debra Kazmerzak, BASW \\ Sheryl Pruin, RN, BSN \\ Wendy Barbaris, $R \mathbf{N}$ \\ Thad E. Abrams, MD, MS
}

\begin{abstract}
Many rural veterans receive care in community settings but could benefit from VA services for certain needs, presenting an opportunity for coordination across systems. This article details the Collaborative Systems of Care (CSC) program, a novel, nurse-led care coordination program identifying and connecting veterans presenting for care in a Federally Qualified Health Center to $V A$ behavioral health and other services based upon the veteran's preferences and eligibility. The CSC program systematically identifies veteran patients, screens for common behavioral health issues, explores VA eligibility for interested veterans, and facilitates coordination with VA to improve healthcare access. While the present program focuses on behavioral health, there is a unique emphasis on assisting veterans with the eligibility and enrollment process and coordinating
\end{abstract}

Address correspondence to M. Bryant Howren, PhD, MPH, Department of Behavioral Sciences \& Social Medicine, College of Medicine, Florida State University, 1115 W. Call Street, Tallahassee, FL 32306, USA. Email: matthew.howren@med.fsu.edu.

M. Bryant Howren, PhD, MPH, VA Office of Rural Health (ORH), Veterans Rural Health Resource Center-Iowa City, Iowa City VA Health Care System, Iowa City, IA, USA.

Thad E. Abrams, MD, MS, VA Office of Rural Health (ORH), Veterans Rural Health Resource Center-Iowa City, Iowa City VA Health Care System, Iowa City, IA, USA.

M. Bryant Howren, PhD, MPH, Florida Blue Center for Rural Health Research \& Policy, College of Medicine, Florida State University, Tallahassee, FL, USA.

M. Bryant Howren, PhD, MPH, Center for Access Delivery Research \& Evaluation (CADRE), Iowa City VA Health Care System, Iowa City, IA, USA.

Thad E. Abrams, MD, MS, Center for Access Delivery Research \& Evaluation (CADRE), Iowa City VA Health Care System, Iowa City, IA, USA.

Debra Kazmerzak, BASW, Iowa Primary Care Association, Urbandale, IA, USA.

Sheryl Pruin, RN, BSN, Community Health Centers of Southeastern Iowa, West Burlington, IA, USA.

Wendy Barbaris, RN, Community Health Centers of Southeastern Iowa, West Burlington, IA, USA.

Thad E. Abrams, MD, MS, Carver College of Medicine, The University of Iowa, Iowa City, IA, USA.

Journal of Behavioral Health Services \& Research, 2022.50-60. (C) 2021 This is a U.S. government work and not under copyright protection in the U.S.; foreign copyright protection may apply. DOI 10.1007/s11414-021-09758-0 
additional care tailored to the patient. As VA expands its presence in community care, opportunities for VA-community care coordination will increase, making the development and implementation of such interventions important.

\section{Introduction}

There are approximately 4.7 million veterans living in rural and highly rural areas of the USA, 2.7 million of which are enrolled in Veterans Health Administration (VA) care. ${ }^{1}$ These veterans tend to be older and are often medically complex, with many experiencing both behavioral and physical health comorbidities. ${ }^{2,3}$ In particular, behavioral health burden is expected with grow in light of the financial challenges and social isolation associated with the COVID-19 pandemic. Such complex patients require more frequent and continuous care, yet many rural veterans face barriers to high-quality healthcare including lack of health insurance, fewer access points, and decreased transportation options, among others. ${ }^{4,5}$ Greater than $60 \%$ of rural Americans also live in behavioral health professional shortage areas. ${ }^{6}$

Many veterans choose to get some or most of their care in community settings, ${ }^{7}$ which has been increasingly a focus in VA the past several years. Examples of expanded access to care in community settings include implementation of VA telehealth programs such as Advancing Telehealth through Local Access Stations (ATLAS) or through legislative acts like the Veterans Choice Act of 2014 or the recently implemented Maintaining Internal Systems and Strengthening Integrated Outside Networks (MISSION) Act of 2018..$^{8-10}$ These initiatives establish partnerships with community-based healthcare organizations to deliver care for veterans meeting certain criteria (e.g., distance to a VA point of care or extensive wait times). These community partners are valued as part of a high performing network of care.

Federally Qualified Health Centers (FQHCs) are one community-based option which veterans may utilize; ${ }^{11}$ in 2019 , nearly 400,000 veterans were seen in these clinics. ${ }^{12}$ FQHCs serve as a safety net provider with the mission of ensuring access to care for underserved populations which may include veterans. Moreover, because the Health Resources and Services Administration (HRSA) requires FQHCs to report annually via the Uniform Data System (UDS) the number of veteran patients seen who have been discharged from uniformed service, ${ }^{13}$ these clinics thus represent an ideal setting to identify veterans and then develop and implement care coordination initiatives with VA, which has been identified as a national priority to improve the quality of health care by the Institute of Medicine. ${ }^{14}$

Care coordination encompasses several levels of communication and cooperation, which include information sharing between providers and systems, knowledge of available resources across systems, and the involvement of multiple stakeholders from patients to interdisciplinary professionals and support staff. ${ }^{15}$ Optimally coordinated care is related to improved quality and outcomes; reduced duplication of services, decreased polypharmacy, and increased patient satisfaction with the care experience have all been documented. ${ }^{16,17}$ Some efforts have focused on patients receiving care in more than one system, ${ }^{17,18}$ known as dual users, whom studies have shown to be associated with a host of poor outcomes, ${ }^{19-22}$ highlighting the need to develop feasible care coordination initiatives to improve VA-community care delivery. ${ }^{23}$ These initiatives may also provide opportunities to improve workforce training regarding veterans issues including behavioral health and community reintegration.

Taken together, the coordination of care for veteran patients receiving community-based care is important and has implications for cross-system healthcare access and delivery as well as workforce training and development. It may also provide an opportunity to better understand how 
veterans use both types of care and avenues for maximizing resources addressing similar issues in care. Because community-based clinics are often under-resourced, collaborations between VA and the community may provide opportunities to lessen the care burden for some clinics, particularly in areas which VA has substantial provider and infrastructure (i.e., telehealth) capacity such as behavioral health.

The present manuscript details a partnership between the VA Office of Rural Health, the Iowa City VA Health Care System, and the Community Health Centers of Southeastern Iowa (CHC/ SEIA) in the development and implementation of a novel, nurse-led care coordination initiative aimed at improving the identification of veterans receiving community care, screening them for behavioral health issues, and subsequently assisting with access to behavioral healthcare and additional services in the veteran's setting of choice. While the present program primarily focuses on behavioral health need, it is unique in its emphases on assisting veterans with the VA eligibility and enrollment process and coordinating additional care tailored to the individual patient. This work is described using the Standard for Quality Improvement Reporting Excellence (SQUIRE) 2.0 guidelines. ${ }^{24} \mathrm{~A}$ human subjects research determination was submitted to the University of Iowa/VA's Institutional Review Board; the project was deemed quality improvement (QI) and thus did not require IRB approval.

\section{Methods}

\section{Program overview and aims}

The Collaborative Systems of Care (CSC) program is a novel nurse-led care coordination program which has demonstrated success in addressing behavioral health needs of rural veterans presenting for care in a large, rural-serving FQHC and bridging an organizational healthcare divide for veterans interested in receiving care from VA. The program's aims are as follows: (1) screen all patients presenting for care at CHC/SEIA for veteran status using a standardized methodology; (2) screen all patients for the following behavioral health issues: depression, anxiety, substance use disorder, and post-traumatic stress disorder (PTSD); (3) identify and assist interested, eligible veteran patients with accessing VA enrollment and care services; (4) ensure veteran patients screening positive for behavioral health issues receive timely, coordinated behavioral healthcare at a VA facility, the CHC/SEIA, and/or other healthcare settings; (5) secondary to behavioral health, provide coordination of other VA services as warranted and requested by the veteran. Key outcomes for this pilot were the number of veterans identified presenting for care at the FQHC, number of veterans screened for behavioral health issues, number of veterans connected to VA care, number of veterans receiving care coordination for behavioral health and/or additional care services, and patient satisfaction with the program.

\section{Setting and pre-implementation}

In 2016, the VA's Office of Rural Health Resource Center-Iowa City began collaborative meetings with representatives of the Iowa Primary Care Association (IPCA) about partnering around veterans issues in community care. There was an immediate recognition that behavioral health needs were paramount and the decision was made to focus on improving behavioral health screening and access to treatment. This was due in part to the continued emphasis within VA on veterans behavioral health needs and the opportunity to shift some behavioral healthcare from an under-resourced FQHC setting to VA, where investments in behavioral health capacity have been significant over the past decade, thereby improving access to care opportunities for both veterans and non-veterans alike. The IPCA facilitated identification of an FQHC community partner to pilot the program, which is now referred to as the CSC. 
Our FQHC partner, the Community Health Centers of Southeastern Iowa (CHC/SEIA) is a Federally Qualified Health Center serving approximately 18,000 unique patients across one primary clinic and three satellite clinics; approximately $5 \%$ of adult patients seen at CHC/SEIA are known military veterans. ${ }^{12}$ One key reason that CHC/SEIA was chosen is because the Southeast region of Iowa is considered a gap area in terms of geographic access to VA facilities; census data has identified nearly 10,000 veterans living in this area. The closest VA clinics are all more than a 60-min drive away. Thus, it was decided that CHC/SEIA represented a prime location to begin this pilot.

The census data also underscored the widely held belief that, at the time the project began, veterans were being substantially undercounted in FQHCs across the country using the standard guidance for capturing veteran status previously outlined in the Uniform Data System (UDS) manual, ${ }^{25}$ which serves as the formal document for annual FQHC reporting requirements. As a result, the first phase of the project focused on improving the identification of veterans in $\mathrm{CHC} /$ SEIA, which began with standardizing the workflow process at intake, updating the item used to request veteran status, and integrating this process into the existing electronic medical record (EMR). This process has been described elsewhere and resulted in a near ten-fold increase of identified veterans in this FQHC, from approximately 50 veterans before implementation to over 500 after. $^{26}$ These results led to specific policy changes regarding the collection of veteran status in FQHCs nationwide beginning in 2020, which are now described in the most recent 2020 UDS reporting manual. ${ }^{13}$

\section{Care coordination processes}

Training and VA integration Once the veteran screening status process was updated and implemented, the focus shifted to establishing care coordination processes, beginning with CHC/SEIA staff training and interaction with our identified VA psychiatrist clinical champion, who served as a liaison from VA to the FQHC. Our program began by securing a portion of effort from an existing FQHC nurse case manager to serve as a care coordinator, which was later expanded to two nurse case managers to handle demand inherent in conducting this program and provide coverage for the main and satellite clinics affiliated with this FQHC. Delivering training related to general veteran issues and specific VA processes was important for clinical and administrative staff awareness, establishing support for the project, facilitating sensitivity to and awareness of unique veteran issues, and understanding nuances of VA care delivery processes. These trainings were fairly extensive and included an all-staff training to orient CHC/SEIA employees to the project in addition to specific, focused trainings as follows: (1) education on military and veteran culture, (2) suicide prevention training specific to veteran needs, (3) VA eligibility training with a special section on VA MISSION, (4) VA electronic medical record training, and (5) shadowing at a VA facility to understand workflow processes within VA. Representatives from several Iowa City VA Health Care System offices and service lines delivered or assisted with these trainings.

As part of this, both nurse care coordinators were credentialed as VA without compensation (WOC) employees. The WOC appointment is authorized by 38 USC 7405 and may be used by VA to employ individuals to do VA work connected with research or QI. A WOC is a federal employee for all purposes with the exception of salary and benefits. This crucial step allows CSC nurses to access VA patient records for the purposes of coordinating care. It also serves to provide these nurses a tangible status within VA which is 
functionally important when communicating with other VA employees during the process of care coordination.

Selection of screening tools To initiate behavioral health screenings for depression, anxiety, substance abuse, and PTSD, FQHC behavioral health practices were reviewed and compared to standard VA practices. Existing tools in use at CHC/SEIA were the 9-item Patient Health Questionnaire for depression (PHQ-9), the Generalized Anxiety Disorder 7-item scale (GAD-7), the Alcohol Use Disorders Identification Test (AUDIT), and the Drug Abuse Screening Test (DAST) as part of the Screening, Brief Intervention and Referral to Treatment (SBIRT) program. ${ }^{27-30}$ Because there was no standard PTSD screener in use, the PTSD Symptom Checklist for DSM-5 (PCL-5) was added and incorporated into the FQHC EMR. ${ }^{31}$ These tools were standardized for all veteran patients to align with VA protocols in terms of screening intervals and follow-up procedures.

Clinic workflow A workflow was created to model the veteran patient path after identification at intake through assistance with eligibility/enrollment, behavioral health screening and follow-up, and care coordination between VA and the FQHC if warranted. All veterans are asked about interest in pursuing VA care and previous history with VA care. For those interested, a limited release of information is signed which allows CHC/SEIA to check for and review existing VA records for the purposes of coordinating care. Irrespective of the screening outcome on behavioral health measures, non-behavioral health needs are assessed. Figure 1 details the complete list of steps in CSC care coordination, which are tailored to each patient, emphasizing veteran choice throughout the process.

The range of care coordination per patient spans several degrees of complexity; after screening for veteran status, it may be limited in scope if the patient indicates no interest in pursuing VA care or knows him/herself to be ineligible. By comparison, a newly identified veteran may indicate interest in VA care, receive eligibility and enrollment assistance if needed, be screened for behavioral health issues, and be scheduled for VA care face-to-face or via telehealth. It is important to note that a veteran electing not to pursue VA care would not alter the level of care received at the FQHC, including the identification and management of behavioral health issues and immediate care delivery for urgent needs. All veterans determined to be ineligible for VA benefits and/or registering negative behavioral health screens are still assessed for non-behavioral health needs and flagged in the veteran care tracking tool for further monitoring or follow-up as indicated by care coordinator judgment. CSC nurses track all veterans identified over time as VA eligibility rules shift and income levels change for veterans so that at future dates veteran eligibility may be revisited if indicated per veteran choice or per clinical judgment. All activity is documented in the EMR and relevant information recorded in the tracking tool (described below). Knowledge of veteran status allows CSC's nurse care coordinators to inquire about salient veterans issues and connect the patient to needed care and services as a function of this status.

Patient care tracking Nurse care coordinators serve each veteran patient, whether the patient remains within CHC/SEIA only or elects care across systems; a personalized care coordination plan is developed for all veterans presenting for care in CHC/SEIA. A veteran care tracking tool was created to record behavioral health screens and corresponding scores, veteran eligibility and enrollment statuses, number receiving behavioral health treatment and location, number actively managed across systems, and services utilized in order to assess the impact of 


\section{Figure 1}

Nurse care coordinator veteran care workflow

Nurse care coordinator veteran care workflow

Step 1. Every unique patient engaging in CHC/SEIA care is screened for veteran status in the medical record. This action occurs prior to steps 2-10, so it should be assumed that all patients impacted by this workflow have already been identified as veterans.

Step 2. Heartland Network (EMR Vendor) provides a daily report of veterans with scheduled appointments for the upcoming 30 days and sends list to Nurse Care Coordinators at West Burlington and Keokuk via secure email.

Patient number, date of appointment, clinic location

Step 3. Prior to appointments, Nurse Care Coordinator reviews CHC/SEIA and VA charts for indicated (e.g., annually or per Nurse Care Coordinator judgement) Behavioral Health (BH) screenings and other information (if relevant).

Nurse Care Coordinator meets with veteran, explains BH screenings, VA care options, coordination, and confirms veteran status

Step 4. Eligibility and VA enrollment opportunities are explored with veteran and/or confirmation of VA benefits established if veteran is interested in assistance. Release of information signed (enabling VA records review and healthcare utilization analyses)

Veteran eligibility screen performed, initial eligibility determination and veteran eligibility and/or enrollment status documented in CHC/SEIA EMR

Step 5. If no VA benefits established, Nurse Care Coordinator assists veteran with securing VA enrollment per veteran choice. Secures copy of DD214 (verification of eligible service record including branch, service era, combat experience, active/reserve duties)

Assists in the completion of 10-10EZ or 10-10EZR (applications for VA health benefits), and SF-180 (request for military records) Faxes completed forms to National Personnel Records Center

Verifies enrollment status with local VA eligibility and enrollment office

Step 6. Nurse Care Coordinator performs clinically indicated BH screens for all veterans regardless of VA eligibility/enrollment status. Screening, Brief Intervention and Referral to Treatment (SBIRT)

- If positive on SBIRT, then additional AUDIT or DAST is performed

- $\quad$ BH screens are done as indicated including PTSD (PCL-5), depression (PHQ-9), anxiety (GAD-7)

Step 7. Nurse Care Coordinator discusses veteran needs including BH positive screens and non-BH needs.

- Veteran preferences for care coordination and preferences for care location discussed and documented

Step 8. Nurse Care Coordinator determines veteran desire to engage in VA care if eligible/enrolled and determines urgency, refers to VA BH care team if BH screens are positive and VA care is desired.

Step 9. Nurse Care Coordinator refers to $\mathrm{CHC} / \mathrm{SEIA}$ BH care team as indicated by veteran choice and/or if veteran is determined to be ineligible for VA, the Nurse Care Coordinator acts on positive BH screens as indicated by level of urgency and discusses resources through Veteran Service Organizations (VSO) or other local resources.

Step 10. Documentation of care coordination for veteran referrals to VA noted in EMR and tracking tool for CHC/SEIA team review and to monitor for follow-up needs.

the intervention relative to its stated aims. Several of these data elements are being incorporated into the clinic EMR.

\section{Results}

In calendar year 2019, 555 unique veterans were identified through intake screening of veteran status upon presentation at CHC/SEIA (Table 1). The mean age of those patients was 64.0 years $(\mathrm{SD}=15.5), 99.1 \%$ were male, and $73.0 \%$ were White. All veterans who met with CSC nurses were offered behavioral health screening, of which 315 of $555(56.8 \%)$ accepted. Of those screened, 60 patients $(19.0 \%)$ not previously receiving behavioral healthcare were determined to be in need of and accepted treatment for behavioral health issues; 19 of 60 patients $(31.7 \%)$ were connected to VA care to initiate treatment at their request.

One hundred thirty-seven of 315 veterans (43.5\%) expressed interest in VA care coordination; $134(42.5 \%)$ were interested in medical (primary and/or specialty) care, $114(36.2 \%)$ were interested in pharmacy, $57(18.1 \%)$ in behavioral health, and $22(7.0 \%)$ in vision services. One hundred fifteen veterans $(36.5 \%)$ have been assisted with the eligibility and enrollment process, with $55(17.5 \%)$ being newly enrolled in VA care. One hundred twenty-five (39.7\%) are actively using both VA and community care (which includes some previously enrolled in VA).

In early 2020, questions regarding patient satisfaction began to be collected. Seven items assessing satisfaction with services offered, friendliness/helpfulness of the nurse care coordinators, importance of the nurse care coordinators, ability to obtain behavioral health or other medical 


\section{Table 1}

Summary of CSC care coordination outcomes 2019

\begin{tabular}{|c|c|c|}
\hline \multirow{3}{*}{ Veterans identified $(N=555)$} & M Age (SD) & $\%$ Male \\
\hline & $64.0(15.5)$ & 99.1 \\
\hline & $\mathrm{N}$ & $\%$ \\
\hline Accepted behavioral health screening ${ }^{1}$ & 315 & 56.8 \\
\hline Accepted behavioral health treatment ${ }^{2,3}$ & 60 & 19.0 \\
\hline Requested care coordination ${ }^{2,4}$ & 137 & 43.5 \\
\hline Behavioral Health & 57 & 18.1 \\
\hline Medical & 134 & 42.5 \\
\hline Pharmacy & 114 & 36.2 \\
\hline Vision & 22 & 7.0 \\
\hline Uses VA \& FQHC ${ }^{2}$ & 125 & 39.7 \\
\hline Assisted w/ eligibility \& enrollment ${ }^{2,5}$ & 115 & 36.5 \\
\hline Newly enrolled in VA Care ${ }^{2}$ & 55 & 17.5 \\
\hline
\end{tabular}

Note. CSC Collaborative Systems of Care; ${ }^{1}$ denominator $=555$ and refers to all veterans identified at $\mathrm{FQHC}$;

${ }^{2}$ denominator $=315$ and refers to all patients who accepted behavioral health screening; ${ }^{3}$ number does not include those already receiving behavioral health treatment; ${ }^{4}$ patients may have requested more than one type of service; ${ }^{5} 115$ were assisted with this process including some who were ultimately ineligible and some previously enrolled but inquiring about eligibility status changes

appointments as a result of the program, and recommendation of the program to others are being collected. Specific questions include "Do you feel that the services offered by our VA Care Coordinator are helping to ensure that your healthcare needs as a veteran are being met?" and "How important is it to have a VA Care Coordinator available at your local doctor's office?" Although the sample is small at this time $(N=37)$, veterans overwhelmingly find value in this program: $94.5 \%$ strongly agreed or agreed that the program is helping to ensure veteran healthcare needs are being met, that the nurse care coordinators listen and are friendly/helpful, that it is important to have these nurses present at the FQHC, and that they would recommend the program to others. The need for behavioral and/or other medical appointments did not apply to all respondents, but the vast majority also strongly agreed or agreed (behavioral health: $95.4 \%$; other medical: $96.5 \%$ ) that the program was helpful in obtaining appointments when they wanted them, with only one respondent to each item indicating disagreement.

\section{Discussion}

The present VA-community care coordination pilot program has shown promise in identifying a substantial number of veterans and coordinating care between an FQHC and VA medical center. The CSC program is particularly important for rural veterans who may benefit most from crosssystem care coordination due to their complex care needs. In our pilot program with one ruralserving FQHC, approximately $60 \%$ accepted behavioral health screening, over $40 \%$ requested VA care coordination, and over one-third have been assisted with the VA eligibility and/or enrollment.

The CSC program is notable for several additional reasons. First, it has assisted with the VA eligibility and enrollment process for veterans who likely would not have pursued VA care or were previously enrolled but unaware of eligibility status changes. Although VA has focused on community care efforts in recent years, policy initiatives like VA's MISSION Act require a veteran to be enrolled in VA care, a process which may be cumbersome for some veterans. This program therefore has contributed to expanded care opportunities for rural veterans that otherwise would not 
have been realized. Second, the availability of VA telehealth has helped beyond delivering behavioral health services to some rural veterans. Due to the COVID-19 pandemic, many face-toface appointments have been cancelled to limit interaction and blunt the spread of disease. The CSC program has connected veterans to VA by telehealth for demonstrations of durable medical equipment, for example, thereby removing an additional face-to-face encounter for veterans who are at increased risk of COVID-19 complications. Aside from the more acute benefit during the COVID-19 pandemic, VA's telehealth infrastructure is expansive and should be considered as a means to improve access more generally for all types of services in VA-community care partnerships. Third, the program has assisted veterans in behavioral health crisis and facilitated coordination of VA inpatient admissions which is directly attributable to the presence of VAconnected nurse care coordinators who have spent much time developing relationships with veterans seen at $\mathrm{CHC} / \mathrm{SEIA}$. Fourth, this program has helped maximize behavioral health resources for both veteran and non-veteran patients. VA has considerable behavioral health resources and policy mandates regarding wait times for mental health. Given that some veterans presenting at CHC/SEIA have chosen to receive VA behavioral healthcare, this presumably frees up additional times for other patients at CHC/SEIA in need of this care. Lastly, CSC nurse care coordinators assist in other ways such as setting up VA care appointments and liaising with VA's Care in the Community (CITC) representatives on behalf of veterans. This VA CITC contact represents an important relational coordination component which has been identified as key to cross-organization coordination; ${ }^{32}$ in this pilot, it has contributed to the reduction of patient burden in tangible ways such as removing the need to miss work to attend to care management logistics. As VA develops its own models of coordination for community-purchased care led by the Office of Community Care, ${ }^{33}$ perhaps there are opportunities to assimilate aspects of the current program to improve coordination between VA and the community, whether the veteran presents in VA or a communitybased care setting.

The CSC program has also served other important roles in advancing care for veterans more generally. In particular, FQHC providers have been part of several VA trainings which underscore the unique needs of veteran patients. This is especially important given evidence which suggests that community providers want knowledge/training on veterans issues and are often lacking in it. $^{34-36}$ For example, surveys of community-based providers have indicated that $84 \%$ reported no direct military experience and only $13 \%$ reported a basic level of military cultural competence. ${ }^{34,35}$ In another study of community-based providers from both primary and specialty care, Fredricks and Nakazawa reported that providers felt uncomfortable addressing routine issues related to the veteran experience. ${ }^{36}$ Similarly, in a sample of community-based primary care providers, Vest and colleagues reported that many failed to ask about veteran status, were unsure of conditions and stressors most likely to impact veterans, and had little knowledge regarding support services available to veterans. ${ }^{37}$ These issues are important irrespective of VA eligibility and knowledge of them may enhance care for all veterans. ${ }^{38}$

It is important to consider the implications of this program in the broader context of dual use of healthcare services. Studies suggest that upwards of $50 \%$ of veterans are dual users of VA and nonVA healthcare and over half have a trusted provider outside of VA. ${ }^{39-41}$ Some studies also indicate that, unsurprisingly, those who live further distances from VA care are more likely to be dual users, suggesting that a substantial portion of rural veterans may be so. ${ }^{42}$ Nearly $40 \%$ of veterans identified in this program were dual users which has been associated with fragmentation, duplication, less treatment for some conditions, and other poor outcomes. ${ }^{19-22}$ The current program was designed in part to track use of other services in addition to behavioral health across both systems, which has likely reduced some duplication and fragmentation as well as reduced wait times for needed services. Indeed, 21 of 22 patient respondents to our satisfaction survey who were in need of specialty behavioral health services, and 28 of 29 who were in need of other VA services, obtained appointments for these services when wanted. Regarding behavioral health 
specifically, the ability to obtain VA appointments when needed may be connected in part to VA's mandated wait times of 30 days or less for outpatient behavioral health services which is less than in some non-VA settings, ${ }^{43}$ including in (rural) mental health professional shortage areas. As the program continues, there will be opportunities to analyze data from each system's EMR which may speak to reductions in duplicative services over time as well as continued management and improvement of behavioral health conditions.

The CSC program is not without limitations. This program has taken place in partnership with a VA medical center with an existing research service. Administrators affiliated with this service line have facilitated WOC appointments for the program's nurse care coordinators, and it is unclear if WOC appointments may be obtained without these administrative connections. In addition, VA's Office of Rural Health provided funds for two FTE nurse care coordinators which likely represent the biggest challenge to replication of this model in other systems of care. Moreover, this pilot program partnered with only one large, rural-serving FQHC without a nearby VA point of care. This makes it difficult to estimate the FTE required for other community-based clinics should this project be scaled up to other sites. Future research should explore low-touch options for connecting veterans presenting in community care settings to VA services, including eligibility and enrollment. Of note, VA support staff have been embedded in community care settings in some instances, ${ }^{44}$ which may be worth examining in clinics serving a large number of veterans. Finally, research demonstrates that patient portals are a valuable tool for those who are dual users, allowing patients to actively participate in information sharing and communication with providers. ${ }^{45}$ Future work should incorporate health informatics tools to foster patient engagement in the care coordination process.

\section{Implications for Behavioral Health}

The CSC program has shown success in improving access and coordination for rural veterans presenting for care in a single FQHC. This program also honors patient preferences regarding where care is received, allowing for greatest continuity of established patient-provider relationships. It represents one of the few successful collaborations to date originating from the community care side of a VA-community care partnership. With respect to behavioral health services, this program has focused on veterans which are an underserved population with relatively high behavioral health needs. For those patients residing in rural areas, systematic screening and connection to care services (in some cases, via telehealth) may have a considerable impact on access to behavioral healthcare and may result in improved outcomes. This is particularly relevant given that many rural areas also are behavioral health professional shortage areas. Moreover, the program has systematically aligned the screening process between VA and our partner FQHC. This includes standardizing behavioral health screening tools, protocols, and screening intervals, which facilitate behavioral healthcare coordination across systems. As a result, this program may inform future attempts to systematically connect VA and community partners around care coordination and may be useful for serving rural, complex patients with limited access to comprehensive healthcare, specifically with respect to behavioral health services. As VA continues to expand its presence in community care collaboration and delivery, it is likely that these opportunities will increase over time, making the development and implementation of VAcommunity care coordination interventions vitally important.

Funding This work was supported in part by an award from the VA Office of Rural Health, Veterans Rural Health Resource Center-Iowa City (VRHRC-IC), Iowa City VA Health Care System, Iowa City, IA (Award \#7345, Co-PIs, Howren/Abrams). The views expressed in this article are those of the authors and do not necessarily represent the position or policy of the Department of Veterans Affairs or United States Government. 


\section{Declarations}

\section{Conflict of interest The authors declare no competing interests.}

\section{References}

1. Rural Veterans. Veterans Health Administration Office of Rural Health. https://www.ruralhealth.va.gov/aboutus/ruralvets.asp. Accessed 17 July 2020.

2. Hoerster KD, Lehavot K, Simpson T, et al. Health and health behavior differences: U.S. military, veteran, and civilian men. American Journal of Preventive Medicine. 2012;43: 483-489.

3. Trivedi RB, Post EP, Sun H, et al. Prevalence, comorbidity, and prognosis of mental health among US veterans. American Journal of Public Health. 2015;105:2564-2569.

4. Buzza C, Ono SS, Turvey C, et al. Distance in relative: unpacking a principal barrier in rural healthcare. Journal of General Internal Medicine. 2011;26(Suppl 2):648-654.

5. Gong G, Phillips SG, Hudson C, et al. Higher US rural mortality rates linked to socioeconomic status, physician shortages, and lack of health insurance. Health Affairs. 2019;38:2003-2010.

6. Thomas KC, Ellis AR, Konrad TR, et al. County-level estimates of mental health professional shortage in the United States. Psychiatric Services. 2009;60:1323-1328.

7. Howren MB, Cozad AJ, Kaboli PK. Considering the issue of dual use in Veterans Affairs patients: Implications and opportunities for improved communication and counseling. Health Communication. 2015;30:838-842.

8. US Department of Veterans Affairs, VHA Telehealth Services. Advancing Telehealth through Local Access Stations. Available online: http://vaww.telehealth.va.gov/pgm/atlas/. Accessed 25 July 2020.

9. Veterans Access, Choice, and Accountability Act of 2014. Public Law 113-146. https://www.congress.gov/113/plaws/publ146/PLAW113publ146.pdf.

10. Strengthening Integrated Outside Networks Act of 2018. Public Law 115-182. https://www.congress.gov/115/plaws/publ182/PLAW115publ182.pdf

11. Heisler EJ, Panangala SV, Bagalman E. Health care for rural veterans: The example of Federally Qualified Health Centers. Congressional Research Service, 2013.

12. Health Resources and Services Administration. National Health Center Data. Available online: https://data.hrsa.gov/tools/datareporting/. Accessed 12 Aug 2020.

13. Health Resources and Services Administration, Bureau of Primary Health Care. Uniform Data System Reporting Instructions for Calendar Year 2020 UDS Data. 2020. Available online: https://bphc.hrsa.gov/sites/default/files/bphc/datareporting/pdf/2020-udsmanual.pdf. Accessed 15 July 2020.

14. Adams K, Corrigan JM. Priority areas for national action: transforming health care quality. Washington, DC, The National Academic Press, 2003.

15. McDonald KM, Sundaram V, Bravata DM, et al. Closing the quality gap: A critical analysis of quality improvement strategies (Vol. 7: Care coordination). AHRQ Publication No. 04(07)-0051-7, 2007. Retrieved from http://www.ncbi.nlm.nih.gov/books/NBK44015/. Accessed 25 July 2020.

16. Bodenheimer T. Coordinating care - A perilous journey through the health care system. New England Journal of Medicine. 2008;358:1064-1071.

17. Doyle D, Emmett M, Crist A, et al. Improving the care of dual eligible patients in rural Federally Qualified Health Centers: The impact of care coordinators and clinical pharmacists. Journal of Primary Care \& Community Health. 2016;7:118-121.

18. Miller LB, Sjoberg H, Mayberry A, et al. The advanced care coordination program: A protocol for improving transitions of care for dual-use veterans from community emergency departments back to the Veterans Health Administration (VA) primary care. $B M C$ Health Services Research. 2019;19:1-10.

19. Howren MB, Cai X, Rosenthal G, et al. Associations of health-related quality of life with healthcare utilization status in veterans. Applied Research in Quality of Life. 2012;7:83-92.

20. Pizer SD. What are the Quality Consequences of Medicare-VA Dual Use? [2013 April]. VA HSR\&D Forum. https:// www.hsrd.research.va.gov/publications/forum/Apr13/. Accessed 25 July 2020.

21. Gellad WF. The Veterans Choice Act and dual health system use. Journal of General Internal Medicine. 2015;31:153-154.

22. West AN, Charlton ME, Vaughan-Sarrazin, M. Dual use of VA and non-VA hospitals by veterans with multiple hospitalizations. $B M C$ Health Services Research. 2015;15:431.

23. Cordasco KM, Hynes DM, Mattocks KM, et al. Improving care coordination for veterans within VA and across healthcare systems. Journal of General Internal Medicine. 2019;34(Suppl 1):1-3.

24. Ogrinc G, Davies L, Goodman D, et al. SQUIRE 2.0 (Standards for QUality Improvement Reporting Excellence): Revised publication guidelines from a detailed consensus process. BMJ Quality and Safety. 2016;25:986-992.

25. Health Resources and Services Administration, Bureau of Primary Health Care. Uniform Data System Reporting Instructions for Calendar Year 2018 UDS Data. 2018. Available online: https://bphc.hrsa.gov/sites/default/files/bphc/datareporting/reporting/2018-udsreporting-manual.pdf. Accessed 17 July 2020. 
26. Howren MB, Kazmerzak D, Kemp RW, et al. Identification of military veterans upon implementation of a standardized screening process in a Federally Qualified Health Center. Journal of Community Health. 2020;45:465-468.

27. Kroenke K, Spitzer RL, Williams JBW. The PHQ-9: Validity of a brief depression severity measure. Journal of General Internal Medicine. 2001;16:606-613.

28. Spitzer RL, Kroenke K, Williams JBW, et al. A brief measure for assessing generalized anxiety disorder. Archives of Internal Medicine. 2006;166:1092-1097.

29. Bohn MJ, Babor TF, Kranzler HR. The Alcohol Use Disorders Identification Test (AUDIT): Validation of a screening instrument for use in medical settings. Journal of Studies in Alcohol. 1995;56:423-432.

30. Skinner HA. The Drug Abuse Screening Test. Addictive Behaviors. 1982;7:363-371.

31. Blevins CA, Weathers FW, Davis MT, et al. The Posttraumatic Stress Disorder Checklist for DSM-5 (PCL-5): Development and initial psychometric evaluation. Journal of Traumatic Stress. 2015;28:489-498.

32. Miller C, Gurewich D, Garvin L, et al. Veterans Affairs and rural community providers' perspectives on interorganizational care coordination: A qualitative analysis. Journal of Rural Health. 2020 [epub ahead of print] doi: https://oi.org/10.1111/jrh.12453.

33. Greenstone CL, Peppiatt J, Cunningham K, et al. Standardizing care coordination with the Department of Veterans Affairs. Journal of General Internal Medicine. 2019;34(Suppl 1), S4-S6.

34. Kilpatrick DG, Best CL, Smith DW, et al. Serving those who have served: Educational needs of health care providers working with military members, veterans, and their families. Charleston: Medical University of South Carolina Department of Psychiatry, National Crime Victims Research \& Treatment Center, 2011. Available online: http://deploymentpsych.org/sites/default/files/mc_resources/ Serving\%20Those\%20Who\%20Have\%20Served.pdf. Accessed 15 July 2020.

35. Tanielian T, Farris C, Batka C, et al. Ready to serve: Community-based provider capacity to deliver culturally competent, quality mental health care to veterans and their families. Santa Monica: RAND Corporation, 2014. Available online: http://www.rand.org/ content/dam/rand/pubs/research_reports/RR800/RR806/RAND_RR806.pdf. Accessed 15 July 2020.

36. Fredricks TR, Nakazawa M. (2015). Perceptions of physicians in civilian medical practice on veterans' issues related to health care. Journal of the Amercian Osteopathic Association. 115:360-368.

37. Vest BM, Kulak J, Hall VM, et al. Addressing patients' veteran status: Primary care providers' knowledge, comfort, and educational needs. Family Medicine. 2018;50:455-459.

38. Brown JL. The unasked question. Journal of the American Medical Association. 2012;308:1869-1870

39. Charlton ME, Mengleing MA, Schlichting JA, et al. Veteran use of health care systems in rural states: Comparing VA and non-VA health care use among privately insured veterans under age 65. Journal of Rural Health. 2016;32:407-417.

40. Liu CF, Bolkan C, Chan D, et al. Dual use of VA and non-VA services among primary care patients with depression. Journal of General Internal Medicine. 2009;24:305-311.

41. Huang G, Muz B, Kim S, et al. 2017 survey of veteran enrollees' health and use of health care. Veterans Health Administration; 2017. Available at: https://www.va.gov/HEALTHPOLICYPLANNING/SOE2017/VA_Enrollees_Report_Data_Findings_Report2.pdf. Accessed 25 July 2020.

42. Nayar P, Apenteng B, Yu F, et al. Rural veterans' perspectives of dual care. Journal of Community Health. 2013;38:70-77.

43. Malowney M, Keltz S, Fischer D, et al. Availability of outpatient care from psychiatrists: A simulated-patient study in three U.S. cities. Psychiatric Services. 2015;66:94-96.

44. Benzer JK, Gurewich D, Singer SJ, et al. A mixed methods study of the association of non-veterans affairs care with veterans' and clinicians' experiences of care coordination. Medical Care. 2020;58:696-702.

45. Turvey C, Klein D, Fix G, et al. Blue Button use by patients to access and share health record information using the Department of Veterans Affairs' online patient portal. Journal of the American Medical Informatics Association. 2014;21:657-663.

Publisher's Note Springer Nature remains neutral with regard to jurisdictional claims in published maps and institutional affiliations. 